\title{
Manual timing in physics experiments: error and uncertainty
}

\author{
David A. Faux* and Janet Godolphin \\ Faculty of Engineering and Physical Sciences, \\ University of Surrey, Guildford, GU2 7XH, UK
}

(Dated: November 28, 2018)

\begin{abstract}
Manual digital timing devices such as stopwatches are ubiquitous in the education sector for experimental work where automated electronic timing is unavailable or impractical. The disadvantage of manual timing is that the experimenter introduces an additional systematic error and random uncertainty to a measurement that hitherto could only be approximated and which masks useful information on uncertainty due to variations in the physical conditions of the experiment. A model for the reaction time of a timekeeper using a stopwatch for a single anticipated visual stimulus of the type encountered in physics experiments is obtained from a set of 4304 reaction times from timekeepers at swimming competitions. The reaction time is found to be well modelled by the normal distribution $N\left(\epsilon, \sigma^{2}\right)=N\left(0.11,0.07^{2}\right)$ in units of seconds where $\epsilon$ and $\sigma^{2}$ are the systematic error and variance for a single time measurement. Consistency between timekeepers is shown to be very good. The reaction time for a stopwatch-operated start and stop experiment can therefore be modelled by $N\left(0,0.10^{2}\right)$, assuming that the average reaction time is the same in both cases. This makes a significant contribution to the uncertainty of most manually-timed measurements. This timing uncertainty can be subtracted out of the variation observed in repeat measurements in the real experiment to reveal the uncertainty solely associated with fluctuations in the physical conditions of the experiment.
\end{abstract}




\section{INTRODUCTION}

Physics experiments that rely on the measurement of a time interval would by choice use an automated electronic timing system. For example, a trolley moving down a slope can be set up to activate a light gate at the top of the slope and again at the bottom of the slope. However, in many instances, it is impractical to use automated electronic timing and measurements are undertaken using hand-held stopwatches. School and undergraduate physics laboratories contain many experiments that rely on manual timing. Examples include experiments that measure the oscillation frequency of a pendulum, the time taken for a body to slide or roll down a slope, various experiments to measure terminal velocity most notably ball-bearings falling or bubbles rising through glycerol/water ${ }^{1}$, measurement of precession frequency to determine the dipole moment of a permanent magnet inside a billiard ball, the determination of " $g$ " using a spring, the study of propagation of thermal waves $^{2}$, the draining of a tank under gravity ${ }^{3}$, applications in statistical mechanics ${ }^{4}$, and more.

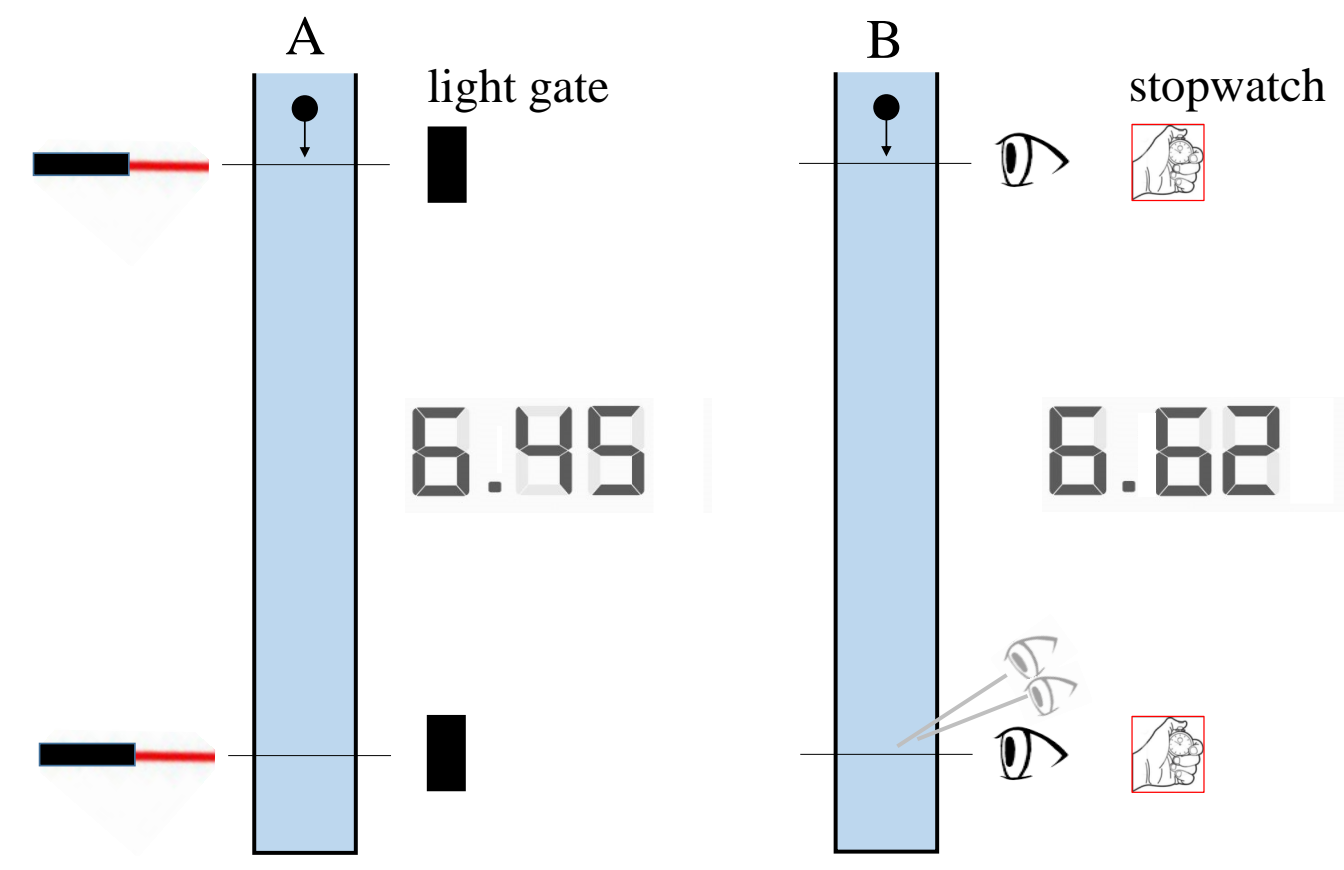

FIG. 1. A schematic diagram in which a ball-bearing is dropped into a tube of glycerol. Terminal velocity is achieved very rapidly and its value is obtained by a measurement of the time taken to move between two markers. Experiments A and B use light gates and a stopwatch respectively to measure the time interval.

The educational value of these experiments is enhanced by a detailed discussion of error 
and uncertainty ${ }^{5}$. Students might discuss the various physical factors that would lead to systematic error and uncertainty in the time measured and their relative importance. Students can be asked to think about human factors that might influence accuracy. How might error and uncertainty be reduced and quantified? And how should a time recorded from a digital stopwatch display be presented if it was to include an uncertainty?

In this article we focus on error and uncertainty associated with manual timing in scientific measurement. We use one experiment to illustrate the issues associated with evaluating and combining the various contributions to the error and uncertainty. The experiment chosen is a "glycerol experiment" widely used both in undergraduate and pre-university laboratories to determine the terminal velocity of a small spherical object, normally a ball-bearing. The terminal velocity can be used to determine the viscosity of the fluid. The glycerol experiment is illustrated in Fig. 1 and provides an excellent example of an experiment in which repeated measurements can readily be obtained, allowing the consideration of a variety of sources of experimental systematic error and random uncertainty. The experiment also lends itself to more ambitious investigation. For example, use of very small spheres allows access to both linear and quadratic drag force regimes ${ }^{6}$, and dropping the ball-bearings at different distances from the tube side leads to predictable differences in terminal velocity ${ }^{7}$.

The article is organised as follows. In Sec. II, sources of error and uncertainty are categorised and discussed. In Sec. III, results are presented for the distribution of human reaction time obtained from a large data source and these are applied to a glycerol experiment with manual timing in Sec. IV. The implication of these results for physics experiments is summarised in Sec. V.

\section{ERROR AND UNCERTAINTY}

We start this section with a summary of key terms and concepts. Formal definitions are found in the Guide to the Expression of Uncertainty in Measurement ${ }^{8}$. A physical quantity which is subject to measurement during experimentation is termed a measurand. Error refers to the result of a measurement (a single measurement or a numerical summary from a series of repeated measurements) minus the true value of the measurand. In practice the true value of a measurand will not be known, even after experimentation. The value of the measurand is estimated from the measurement, or measurements, and the range of values 
within which the true value of the measurand is reasonably believed to lie is characterized by a dispersion parameter, termed the uncertainty. There are two types of uncertainty evaluation. Uncertainty calculations using statistical methods which depend entirely on the data of the experiment are classified as Type A. Such methods only apply to repeated measurements and include: estimation of the uncertainty from the standard deviation of the measurements; uncertainty estimation from fitting a model to data by the method of least squares. Uncertainty evaluations which do not depend entirely on the data of the experiment are classified as Type B. Such evaluations are based on scientific judgement and rely on factors such as: previous data; calibration information; an assumed distribution. For a single measurement, the uncertainty will necessarily be Type B and often depends on the resolution of the measuring device: for example, a vernier calliper might measure a distance correct to $\pm 0.005 \mathrm{~mm}$. When a formula involves measurements of different quantities, the contribution that each measurement makes to the uncertainty can be evaluated with Type A and Type B methods, as appropriate. The combined uncertainty of the calculated quantity can be improved by reducing or eliminating any source of error. Type A and Type B uncertainties can be combined by taking the square root of the sum of the squares of the individual uncertainties.

Where possible, automated timing equipment with light gates is used to measure time intervals in physics laboratory experiments. The moment an object blocks a source of light, timing is started by an internal clock which is then stopped at a second trigger due to the object crossing the second light gate. The advantage of automated electronic timing equipment is that systematic error and random uncertainty associated with the human experimenter are removed. The variability in times obtained from repeat measurements using automated timing therefore represents variations in the physical conditions of the experiment since these physical variations are usually larger than the small variations in the automated timing. For a terminal velocity experiment using glycerol illustrated in Experiment A of Fig. 1, the spread of measured times may be due to changes in the viscosity of the fluid, perhaps caused by temperature changes, distance of the ball-bearing from the side of the container, variations in the number of small bubbles in the glycerol, changes in the condition of the ball-bearing, and so forth.

The two versions of the experiment in Fig. 1 are used to illustrate possible sources of experimental error and uncertainty and issues associated with evaluating these. The first 
experiment, Experiment A, employs automated electronic timing whilst Experiment B uses hand-held stopwatches. In both experiments the objective is to find the terminal velocity of a given ball-bearing using the equation

$$
v=\frac{d}{\tau}
$$

Here $d$ is the distance between two markers, with the highest marker positioned at a depth sufficient to ensure that the ball bearing attains terminal velocity before it reaches the marker, and $\tau$ is the time for the ball-bearing to move the distance $d$. For both experiments it is reasonable to assume that the measurements for $d$ and $\tau$ are independent. Thus, in both cases the uncertainty, $\delta v$, in the terminal velocity, $v$, is given by:

$$
\left(\frac{\delta v}{v}\right)^{2}=\left(\frac{\delta \tau}{\tau}\right)^{2}+\left(\frac{\delta d}{d}\right)^{2}
$$

where $\delta \tau$ and $\delta d$ are the uncertainties in measurements for $\tau$ and $d$.

\section{A. Experiment A}

In version $\mathrm{A}$ of the experiment, $n$ independent repeat measurements, $\tau_{i}$, for $i=1 \ldots n$, are made of the time taken for the ball-bearing to travel between the start and finish markers. In experiment $\mathrm{A}$, the time measurement is made by an automated electronic timing system without human intervention. The purpose of undertaking repeat time measurements is to obtain a satisfactory estimate of $\tau$, the true time taken for the ball-bearing to move between the markers. The variation in the $n$ individual measurements $\tau_{i}$ is due to fluctuations in the

physical conditions of the experiment and these measurements are assumed to constitute a random sample from a normal distribution with mean $\tau$ and standard deviation $\sigma_{1}$, that is the $N\left(\tau, \sigma_{1}^{2}\right)$ distribution. The true values of $\tau$ and $\sigma_{1}$ are unknown. The best estimate of $\tau$ is calculated from the mean of the data as:

$$
\bar{\tau}=\frac{1}{n} \sum_{i=1}^{n} \tau_{i}
$$

The uncertainty in $\tau$ is evaluated by a Type A approach. Using statistical theory, the true unknown time interval $\tau$ is taken to be $\bar{\tau} \pm \sigma_{1} / \sqrt{n}$ to one standard deviation. That is, for 
approximately $68 \%$ of samples of size $n$, the true value of $\tau$ will lie between $\bar{\tau}-\sigma_{1} / \sqrt{n}$ and $\bar{\tau}+\sigma_{1} / \sqrt{n}$. The data provide an estimate of $\sigma_{1}$ via the formula:

$$
\sigma_{1} \approx s_{1}=\sqrt{\frac{1}{n-1} \sum_{i=1}^{n}\left(\tau_{i}-\bar{\tau}\right)^{2}} .
$$

The value of $s_{1}$ is calculated from the data and the measurement of the time interval $\tau$ is presented as $\bar{\tau} \pm s_{1} / \sqrt{n}$. We use $\bar{\tau}$ as the best estimate of $\tau$ in (1) and (2), and $\delta \tau=s_{1} / \sqrt{n}$ as the uncertainty in $\tau$ in (2). With $\sigma_{1}$ being estimated by $s_{1}$, the evaluation of $\delta \tau$ is based entirely on the data of the experiment. At this point some comment should be made on the reliability of $s_{1}$ as an estimate of $\sigma_{1}$ in (3). The fractional uncertainty in $s_{1}$ is $1 / \sqrt{2(n-1)}$ (see for example, Chapter 5 of Taylor ${ }^{5}$ ). Thus, a large number of measurements is required in order for the uncertainty $\delta \tau$ to be reliably assessed.

A measurement of the distance travelled by the ball-bearing during the time interval $\tau$ and an assessment of the uncertainty of this measurement are required to complete the calculation of $v$ and the uncertainty, $\delta v$. The distance, $d$, between the two visual line markers aligned with the light gates is determined by a single measurement using a ruler. For illustration, we take the ruler to be graduated in millimeters. Some judgement is required (forming a useful discussion with students) as to whether the uncertainty in the measurement of $d$ is justifiably $\delta d=0.5 \mathrm{~mm}, 1 \mathrm{~mm}$ or larger. One might argue that thick line markers and recognition of an unknown and systematic error due to the distance difference between the light gates and line markers may be accommodated by the larger uncertainty estimate of $1 \mathrm{~mm}$ or more.

Notice that the method of evaluating the uncertainty $\delta d$ is Type B, being based on assessment of the error magnitude from knowledge of the measuring equipment and apparatus set up. Since $d$ is obtained from a single measurement, Type A uncertainty evaluation, such as a standard deviation calculation of type (3), is not available for $\delta d$. Occasionally, error analysis that fails to recognise the difference between estimates based on one measurement and those based on a set of measurements leads to illogical consequences ${ }^{9}$. In the present experiment, the estimation of $v$ and the error analysis proceeds using the measured value of $d$ and an appropriate value for $\delta d$. These, together with estimates of $\tau$ and $\delta \tau$ already obtained yield values for $v$ and $\delta v$ via (1) and (2).

In assuming that the time measurements $\tau_{i}$ arise from a normal distribution with mean 
$\tau$, any systematic timing errors are taken as being negligible. Nonetheless, systematic error will exist. Most digital timers rely on the time base oscillations of a quartz crystal. The quartz crystal is normally set to oscillate at $32768=2^{15} \mathrm{~Hz}$ so that each "tick" is of duration $3.0518 \times 10^{-5} \mathrm{~s}$. A circuit comprising flip-flop digital dividers counts the number of oscillations between a start signal and a stop signal ${ }^{10,11}$. The number of counts expressed as a binary number is multiplied by $3.0518 \times 10^{-5}$ to obtain a time interval in seconds. Thus the error in the measurement of a time difference would be about $30 \mu \mathrm{s}$. In addition, and more significantly, an error will arise due to the process of converting a time to a form for display presentation. For example, a time of $6.62 \mathrm{~s}$, as in Fig. 1, might be assumed to indicate a measured time between $6.615 \mathrm{~s}$ and $6.625 \mathrm{~s}$. If, however, the digital display is produced as described by the IBM Knowledge Center ${ }^{12}$, the measured time would lie between $6.62 \mathrm{~s}$ and $6.63 \mathrm{~s}$, introducing a small systematic error.

In the context of Experiment A, we will briefly justify that such an error can be considered negligible. Consider a single time observation of $6.62 \mathrm{~s}$ with $d=150 \mathrm{~mm}$. The fractional uncertainty of the time measurement attributable to the systematic timing error is $0.005 / 6.26=7.6 \times 10^{-4}$. By comparison, taking $\delta d=0.5 \mathrm{~mm}$ gives the fractional uncertainty in $d$ as $\delta d / d=3 \times 10^{-3}$. The difference in the magnitudes of the uncertainties is

magnified when they are combined via (2), which yields $\delta v / v=3 \times 10^{-3}$, that is, the same value as the fractional uncertainty in $d$. Thus regardless of the overall magnitude of $\delta \tau$, the contribution of any systematic timing error to the uncertainty $\delta v$ is likely to be insignificant. In general, systematic errors associated with the time measurements in Experiment A are unknown and cannot be estimated and are assumed negligible.

\section{B. Experiment B}

It is often impractical to set up electronic timing systems in which case a hand-held timing device (usually a stopwatch) is used instead. This is Experiment B in Fig. 1. The stopwatch is started by an experimenter the moment the ball-bearing crosses the first mark and stopped when the ball-bearing is seen to cross the second mark. Manual timing introduces additional sources of error and uncertainty. For example, parallax errors, in which the observer identifies the moment the ball-bearing crosses a line by viewing at an angle, can lead to both systematic error and uncertainty. Such errors are generally discussed and acknowledged but are difficult 
to quantify. Moreover, errors of timing due to parallax are likely to be subsumed within the general reaction time variability of the human stopwatch operator. Thus, in Experiment B consideration needs to be given to the error and uncertainty associated with manual timing.

In the following section we provide definitive values for the error and uncertainty of human reaction time associated with an anticipated visual trigger for a single measurement event. This is then extended to encompass the situation of Experiment B in which both start and stop points of a manual time are anticipated by visual cues.

\section{REACTION TIMES}

Human reaction time can be defined as "the time elapsed from stimulus presentation until a reaction/response occurs" 13 . The type of stimulus can fall into one of two categories: an anticipated stimulus or an unanticipated stimulus. In the context of laboratory physics, a stimulus is normally anticipated. For an anticipated stimulus, the motion of an object can be followed by the eye and the moment when the object meets a designated marker can be anticipated in advance. The stopwatch is activated by the hand in response to the eye seeing the object meet the marker. An unanticipated stimulus is one which, by definition, cannot reliably be anticipated in advance. An example of an unanticipated stimulus is the classic reaction time measurement in which a ruler is held at the top end by one experimenter with the bottom end placed between the finger and thumb of a second participant with the finger and thumb about $3 \mathrm{~cm}$ apart. At some point the ruler is released and the participant closes their finger and thumb to grip the ruler ${ }^{14}$. The position of the grip point on the ruler depends on how long it takes the participant to react and therefore the time it takes an unanticipated visual stimulus (the movement of the ruler upon release) to be processed by the brain to elicit a manual response, in this case the closure of the finger and thumb.

We present a statistical analysis of a large volume of data obtained from swimming competitions in Hampshire, UK, during 2016-18. In swimming competitions, electronic timing is started automatically by a signal from the starting box and is stopped when the swimmer presses on a touch-sensitive pad. This gives the automated race time for the respective swimmer. In addition, semi-manual timing provides a back-up time if there is a problem with the electronic timing at the finish of the race, usually a light touch by the swimmer failing to activate the pad sensor. The semi-manual timing starts automatically in 
the same way as the electronic timing. The timekeeper watches the swimmer approach the pad at the conclusion of the race and presses a button at the end of a stick connected to the timing circuitry when they observe the touch. To emphasise, timekeepers are trained to press the button only at the moment they see the swimmer touch the pad. The semi-manual time $t_{s m}$ is recorded automatically together with the automated race time $t_{e}$ and saved to file.

The difference between an automated race time and semi-manual time, $\delta t=t_{s m}-t_{e}$, can be considered as the reaction time of the manual operator in operating the stop mechanism at the end of the race. There may be systematic errors, the most significant of which will be associated with the time difference between the moment the swimmer touches the timing pad (prompting the timekeeper to press the back-up button) and the activation of the pressure sensor. The sensor is activated when a gap of $2 \mathrm{~mm}$ is closed causing a circuit to be completed and the dispatch of a pulse signal to stop the electronic timer. Even the slowest swimmer drifting to finish a race will move at $1 \mathrm{~m} / \mathrm{s}$ and so the maximum time differential between the touch of the pad and sensor activation is about $0.002 \mathrm{~s}$, contributing a small systematic error to $\delta t$.

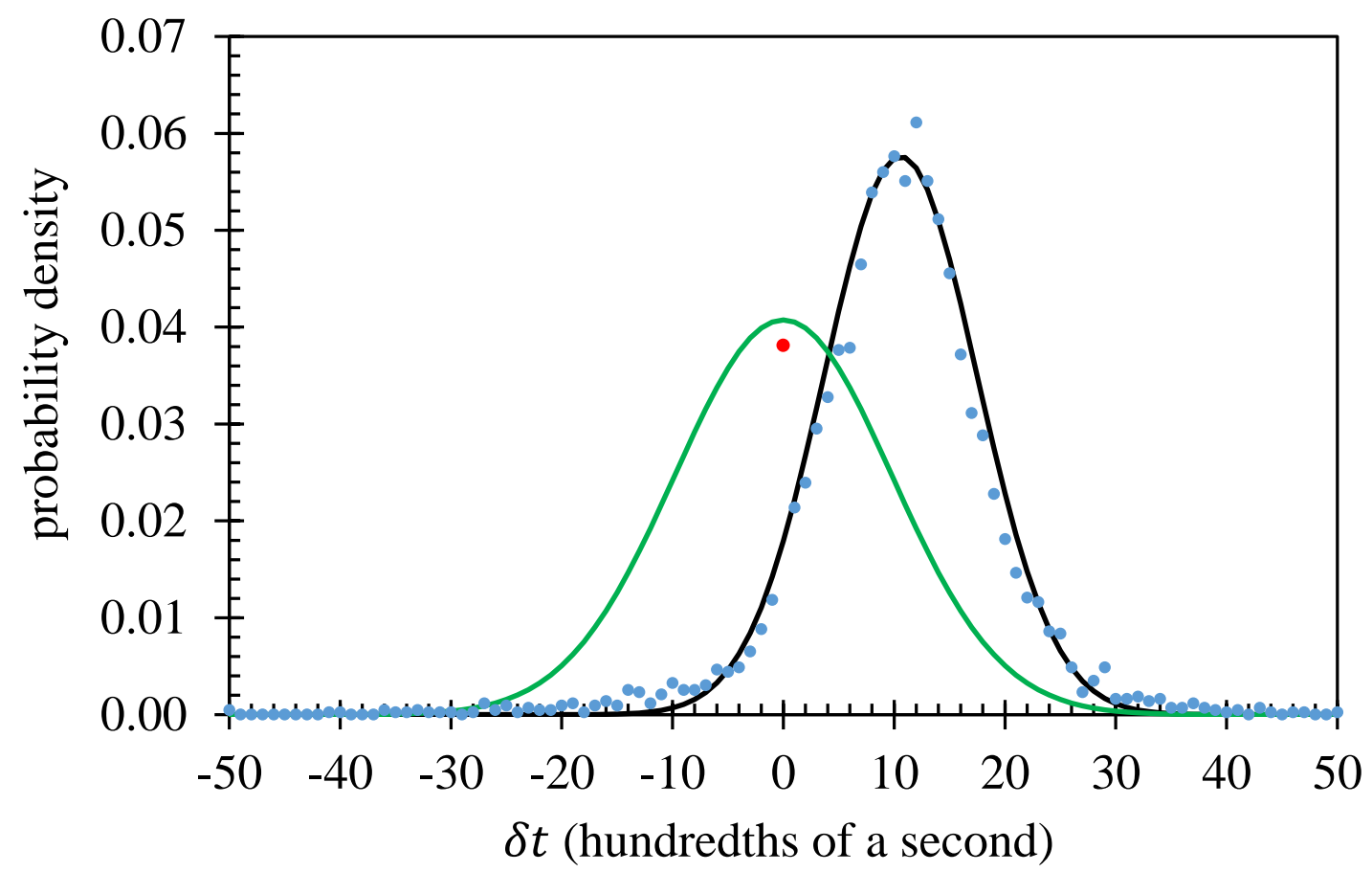

FIG. 2. The distribution of reaction times obtained from 4304 semi-manual times is presented. The normal distribution (black) is fit to the data. The single point in red at $\delta t=0$ is anomalous (see text). The normal distribution (green) is the predicted distribution for fully manual timing. 
Semi-manual and automated race times were obtained from 4304 swims. The differences between the recorded times for each swim give 4304 values of the reaction time, $\delta t$. These are presented in Fig. 2 displayed in units of hundredth of a second. The distribution of reaction times is approximately normal with a positive mean as one would expect. The data are fitted to a normal distribution $N\left(\epsilon, \sigma^{2}\right)$ providing a best fit (obtained by minimising the squared difference) with a mean of $\epsilon=0.106 \mathrm{~s}$ and a standard deviation $\sigma=0.069 \mathrm{~s}$. The data point at $\delta t=0$ is not representative because, on occasion, the automated race time is absent and is replaced by the semi-manual time by the operator. In such cases, the two recorded race times are identical. Hence we cannot distinguish between a semi-manual time substitution and a genuine $\delta t=0$. For this reason, the data point at $\delta t=0$ is omitted from the analysis. These results provide numerical values for the systematic and random uncertainty associated with a single activation of a stop signal prompted by an anticipated visual trigger.

The 4304 values of $\delta t$ are produced by adult timekeepers (age 20-60) who have been trained to press the button only when they observe the swimmer touch the pad. The mean reaction time is found to be $0.106 \mathrm{~s}$ and is due to the time taken for the visual trigger to be processed by the visual cortex in the brain, for the brain to produce a response, for the response signal to travel along the nervous system to the hand and for the muscles in the hand to respond to stop the timer. Various factors and influences are known to impact timekeeper performance such as gender and handedness ${ }^{15,16}$, lifestyle and environmental factors ${ }^{17}$ and stress $^{18}$. A question therefore arises as to the consistency of timekeepers leading to the distribution in Fig. 2. The distribution could reflect timekeepers each with similar characteristics (similar $\epsilon$ and $\sigma$ ), or reflect the amalgamation of a variety of variable individual performances. One swimming competition allowed investigation of this question. The competition yielded 349 values of $\delta t$ distributed amongst seven lanes with each lane having one timekeeper assigned for the entire event. All seven lanes were used for each race resulting in an approximately equal number of values of $\delta t$ for each timekeeper. The mean and standard deviation for each timekeeper are presented in Fig. 3. The performance of the timekeepers is found to be remarkably consistent. Both the average response and variation of response are consistent across individuals in this small sample. These results provide confidence that the response time of a typical person to an anticipated visual trigger can be adequately modelled by the $N\left(0.11,0.07^{2}\right)$ distribution. Timekeeper consistency is also 
found to be good (even between experienced and novice timekeepers) in research studies in the sporting environment, ${ }^{19,20}$ giving justification to the assumption that experimenters will perform in a consistent fashion.

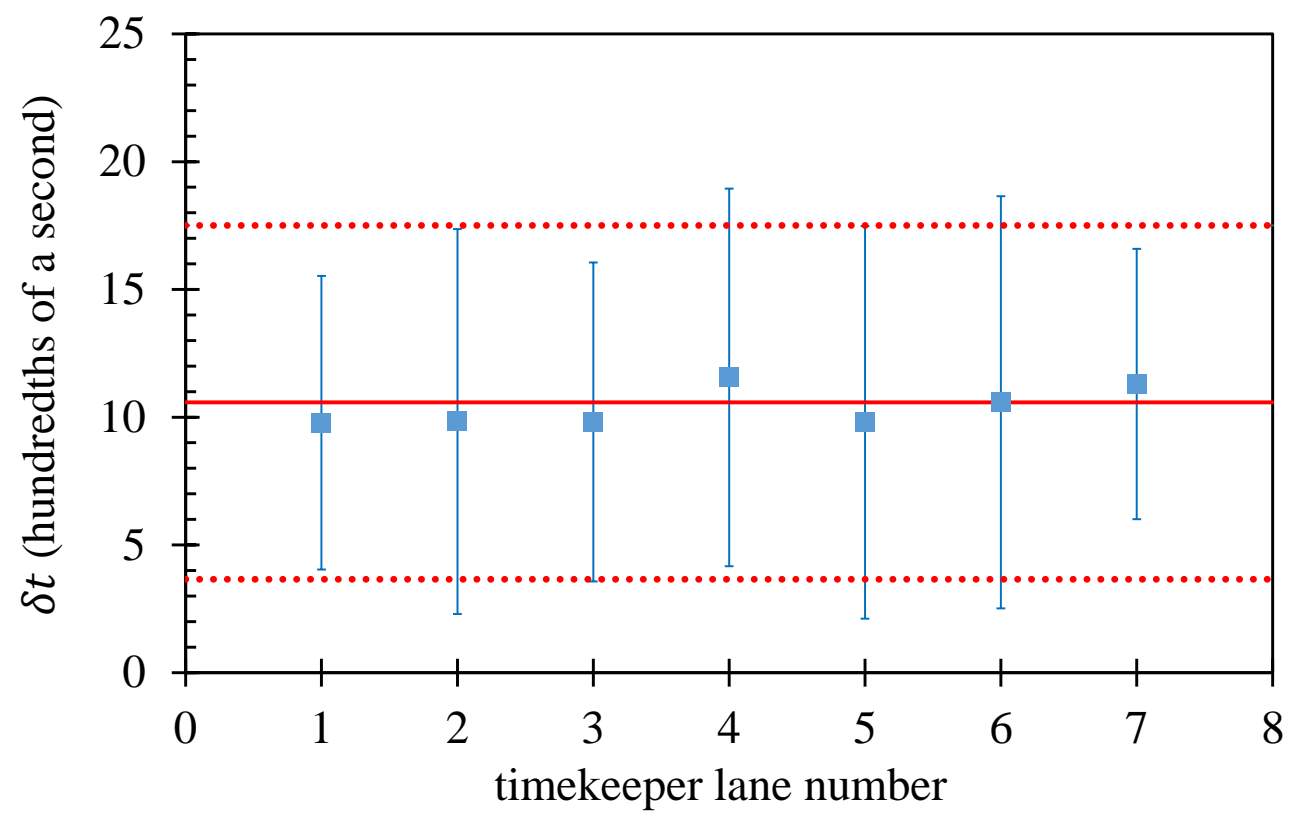

FIG. 3. The mean time delta and standard deviation for individual timekeepers labelled 1-7 are presented. For comparison the horizontal lines represent the mean and standard deviation of the distribution for 4304 time deltas displayed in Fig. 3

There is a vast amount of literature related to human reaction times to various stimuli and considerable interest in the sport conditioning sector but little that provides a direct comparison with the present data, none that provides results from such a large sample and none that is as directly relevant to timekeeping in the laboratory. Welford states that the reaction time is generally accepted to be $0.19 \mathrm{~s}$ for a non-anticipated trigger ${ }^{21}$. A study involving 44 physics students performing the dropped-ruler experiment, described earlier, yielded reaction times of about $0.24-0.27 \mathrm{~s}^{14}$. Here the brain has to (i) respond to the unanticipated movement of the ruler and (ii) close the fingers. Our lower mean reaction time of $0.11 \mathrm{~s}$ for an anticipated stimulus seems plausible.

The error and uncertainty described as the reaction time for a single manual response to an anticipated event presented in Fig. 2 is modelled by $N\left(0.11,0.07^{2}\right)$. This distribution can be used to model the reaction times associated with both the manual start and finish for a timekeeper using a stopwatch to record a time interval with anticipated start and end points. Therefore, for a typical person providing a single response with reaction time characterised 
by $N\left(0.11,0.07^{2}\right)$, the discrepancy between a true time interval and the manual time can be modelled by a normal distribution with mean $0 \mathrm{~s}$ and standard deviation $\sqrt{2} \times 0.07 \approx 0.10 \mathrm{~s}$, that is by $N\left(0,0.10^{2}\right)$. This curve (green) is shown in Fig. 2. In other words, assuming that the reaction times for the manual start and stop are consistent, the distribution of the elapsed time measured by a stopwatch should have mean equal to the true time with standard deviation of about $0.10 \mathrm{~s}$.

\section{APPLICATION TO LABORATORY PHYSICS EXPERIMENTS USING MAN- UAL TIMING}

The $N\left(0,0.10^{2}\right)$ characteristic distribution for the reaction times for a manual start-stop measurement developed in Sec. III is now applied to the analysis of data from a glycerol terminal velocity experiment illustrated in Fig. 1 as Experiment B. There are several reports placed online that include the repeat time measurements obtained using a stopwatch (rather than just their mean). A fine example is due to Patel $^{22}$ who presents a table containing five repeat time measurements for each of eight different ball-bearing radii in their Appendix 2 . The five measurements taken by Patel for a given ball-bearing radius form a random sample from the $N\left(\tau, \sigma_{2}^{2}\right)$ distribution, where $\sigma_{2}$ is the true standard deviation of measurements of $\tau$ which will include variability due to both human reaction time and the physical conditions of the experiment. We would expect $\sigma_{2}>\sigma_{1}$ from electronically-timed experiment A.

Here, as with the calculations for Experiment A, we assume systematic timing errors are negligible. If we momentarily also assume that any variability in the Patel measurements is due solely to the variation in reaction time of the experimenter, then we have a reliable estimate for $\sigma_{2}$ using $\sigma_{2}=\sigma=0.10 \mathrm{~s}$ from the 4304 repeat measurements in Sec. III. Thus the uncertainty in a single time measurement of $\tau$ is $0.10 \mathrm{~s}$ to one standard deviation. Further, with five repeat measurements, the estimate of the time interval $\tau$ is presented as $\bar{\tau} \pm 0.10 / \sqrt{5} \mathrm{~s}$ to one standard deviation. Here, the evaluation of uncertainty is Type B, since the value $\sigma_{2}=0.10 \mathrm{~s}$ has been obtained from the assumed distribution $N\left(0,0.10^{2}\right)$ and not from the data of the experiment. The motivation for the approach is that the measure $\delta \tau=0.1 / \sqrt{5}$ can be considered reliable.

By contrast, the inadequacy of seeking an estimate of $\sigma_{2}$ and thus the uncertainty $\delta \tau$ with a Type A approach by calculating the standard deviation of five repeat time measurements 
is illustrated using the data of Patel ${ }^{22}$. The eight sets of five measurements yield estimates of the standard deviation $\sigma_{2}$ in the wide range 0.07-0.18. Such variation in the estimates is to be expected since the fractional uncertainty in an estimate of $\sigma_{2}$ from a sample of size five is $1 / \sqrt{8}$, that is, about $35 \%$.

It is notable however that the average of the eight estimates for $\sigma_{2}$ is $0.11 \mathrm{~s}$ which is very close to the accurate value of 0.10 s presented in Sec. III. This suggests that the spread of repeat time measurements is dominated by the variation in human reaction time. In other words, $\sigma_{1} \ll \sigma_{2}$. This is not surprising since we would not expect carefully-executed terminal velocity experiments using glycerol to be sensitive to variation in physical conditions during a set of repeat measurements executed over short distances. Variations would be expected to become more significant over longer distances.

The terminal velocity $v$ can now be determined using the smallest ball-bearing radius as an example in which $\bar{\tau}=3.71 \mathrm{~s}$. Using $d=200 \pm 1 \mathrm{~mm}$ and Eq. 2, the result yields $v=53.9 \pm 0.7 \mathrm{~mm} / \mathrm{s}$.

\section{SUMMARY AND CONCLUSIONS}

Many experiments in the physics laboratory rely on manual (stopwatch) timing. The experimenter starts and stops the stopwatch prompted by visual cues. Normally, the visual cues are anticipated, that is the experimenter can see the progress of an object and can anticipate the moment of the cue. For example, a trolley rolls down a slope and the experimenter starts and stops their stopwatch at the moment the trolley passes each of two markers drawn on the slope. We show that the variability of reaction time of the experimenter to the anticipated visual cue can make a significant contribution to the uncertainty of the experiment. Estimating this uncertainty by undertaking repeated timings is unreliable with a small number of repeats that can be undertaken in practice.

We identify timing data obtained from swimming competitions as a reliable source of the mean and variance of a reaction time for an anticipated visual cue of the type encountered in physics experimentation. A set of 4304 reaction times associated with a single visual cue is found to be characterised by a normal distribution with mean $0.11 \mathrm{~s}$ and standard deviation $0.07 \mathrm{~s}$, that is the $N\left(0.11,0.07^{2}\right)$ distribution. Therefore, the timing error produced by an experimenter operating fully-manual timing (manual start, manual stop) is characterised by 
$N\left(0,0.10^{2}\right)$ assuming the same reaction time for both the start and stop processes. Very good consistency between a sample of seven timekeepers added to evidence in the literature indicates excellent consistency in performance between timekeepers in terms of the mean and variance of the reaction time distribution.

The human and non-human contributions to the error and uncertainty are summarised and discussed for an example terminal velocity experiment in which ball-bearings descend through glycerol. Based on an online report containing raw times, we conclude that the contribution to reaction-time uncertainty may dominate the contribution due to the variability of physical conditions of the experiment.

The results for the reaction time uncertainty presented here are useful for the analysis of experiments undertaken in the physics laboratory which employ stopwatch timing. With confidence, the random uncertainty associated with a stopwatch-generated time interval is $0.10 \mathrm{~s}$ at one standard deviation. Likewise, the random uncertainty associated with the mean of $n$ repeat time interval measurements is $0.10 / \sqrt{n}$ s. This is particularly useful for small samples where measures of standard deviation calculated from the data set can be unreliable. The result also enables the reaction-time uncertainty to be subtracted out of the variation observed in repeat measurements in the real experiment to reveal the uncertainty solely associated with variations in the physical conditions of the experiment.

\section{ACKNOWLEDGMENTS}

We are very grateful to officials at Hart Swimming Club, Fleet, Hampshire, UK, who have allowed access to swim race data.

* d.faux@surrey.ac.uk

1 L. Liu, H. Yan, G. Zhao and J. Zhuang, "Experimental studies on the terminal velocity of air bubbles in water and glycerol aqueous solution", Experimental Thermal and Fluid Science 78, $254-265$ (2016);

2 A. Bodas, V. Gandiá, and E. López-Baeza, "An undergraduate experiment on the propagation of thermal waves", Am. J. Phys. 66, 528 (1998); https://doi.org/10.1119/1.18899 
3 J. N. Libii, "Mechanics of the slow draining of a large tank under gravity", Am. J. Phys. 71, $1204(2003)$.

4 J. J. Prentis, "Experiments in statistical mechanics", Am. J. Phys. 68, 1073 (2000).

5 J. Taylor, "Introduction to error analysis, the study of uncertainties in physical measurements" (University Science Books, New York, 1997), Ch. 3.

6 J. P. Owen and W. S. Ryu, "The effects of linear and quadratic drag on falling spheres: an undergraduate laboratory", Eur. J. Phys. 26, 1085-1091, (2005).

7 C. H. Ataíde, F. A. R. Pereira and M. A. S. Barrozo, "Wall effects on the terminal velocity of spherical particles in Newtonian and non-Newtonian fluids", Braz. J. Chem. Eng., 16, 387-394 (1999).

8 BIPM, IEC, IFCC, ISO, IUPAC, IUPAP, OIML, "Guide to the Expression of Uncertainty in Measurement", Geneva, Switzerland: International Organization for Standardization, available at <https://www.bipm.org/utils/common/documents/jcgm/JCGM_100_2008_E.pdf/> (2008).

9 For example, an image suggests a new asteroid has a diameter of $d=2 r=0.5 \mathrm{~km}$ with an uncertainty of $0.2 \mathrm{~km}$. What is its volume? $V=4 \pi r^{3} / 3$ so that $\delta V=4 \pi r^{2} \delta r$ yielding $V=0.065 \pm 0.079 \mathrm{~km}^{3}$.

10 M. Robbins, "Electronic Clocks and Watches", Howard W. Sams Co., Indianapolis, IN, (1975).

11 Jeff C. Gust, Robert M. Graham and Michael A. Lombardi, "Stopwatches and Timer Calibrations", NIST Recommended Practice Guide, (2009 edition) available at <https://tf.nist. gov/general/pdf/2281.pdf/>

12 Converting binary fields to time of day format, IBM Knowledge Center, at <https://www.ibm.com/support/knowledgecenter/en/SSLTBW_2.1.0/com.ibm.zos.v2r1. ieag200/cnvttime.htm/> [accessed March 2018].

13 J. M. Medina, J. A. Daz, and K. H. Norwich, "A theory of power laws in human reaction times: insights from an information-processing approach", Front. Hum. Neurosci., 8, 621 (2014). doi: 10.3389/fnhum.2014.00621

14 C. Saxena, R. Kaur and P. Arun, "Reaction time of a group of physics students", Physics Education 43, 309 (2008).

15 A. Jain, R. Bansal, A. Kumar and K. D. Singh, "A Comparative Study of Visual and Auditory Reaction Times on the Basis of Gender and Physical Activity Levels of Medical First Year 
Students", Int. J. Appl. Basic Med. Res. 5, 124-127, (2015).

16 S. Dane and A. Erzurumluoglu, "Sex and Handedness Differences in Eye-hand Visual Reaction Times in Handball Players", Int. J. Neurosci., 113, 923-929, (2003).

17 H. Nakamoto and S. Mori, "Sport-specific Decision-making in a Go/Nogo Reaction Task: Difference among Nonathletes and Baseball and Basketball Players", Perceptual and Motor Skills 106, 163-170, (2008).

18 A. M. Chandra, S. Ghosh, S. Barman, R. Iqbal and N. Sadhu, "Effect of Exercise and HeatLoad on Simple Reaction Time of University Students", Int. J. Occup. Saf. Ergon. 16, 497-505, (2010).

19 J. L. Mayhew, J. J. Houser, B. B. Briney, T. B. Williams, F. C. Piper and W. F. Brechue, "Comparison between hand and electronic timing of 40-yd dash performance in college football players", J. Strength Cond. Res., 24, 447-451, (2010).

20 J. B. Mann, P. J. Ivey, W. F. Brechue and J. L. Mayhew, "Validity and reliability of hand and electronic timing for 40-yd sprint in college football players", J. Strength Cond. Res., 29, 1509-1514, (2015).

21 "Choice reaction time: Basic concepts" in Reaction times, ed. A. T. Welford, (London; New York: Academic Press, 1980), Ch. 3.

22 "Determination of viscosity of glycerine using falling ball bearing technique", Mitul Patel, available at <http://astrocosmos.weebly.com/uploads/7/8/2/7/7827244/viscosity_report. pdf/> [accessed July 2018]. 